\title{
Types de risques perçus et réducteurs de risques dans le commerce électronique : le cas du site Fnac.com
}

\author{
Christophe Bèzes ${ }^{1}$ \\ Professeur-chercheur permanent Istec* \\ Largepa - Université Panthéon-Assas \\ Click M'Brick Conseil \\ academic@ @lickmbrick.net \\ * Istec, 12 rue Alexandre Parodi 75010 Paris
}

\section{Résumé}

Cette recherche aborde les freins relatifs à l'achat sur Internet, non par le concept de confiance déjà largement mobilisé dans la littérature, mais par celui de risques spécifiques perçus. Réalisée sur 1167 adhérents de la Fnac, elle identifie les types de risques perçus qui pénalisent le plus l'achat en ligne, puis les caractéristiques du site que l'enseigne concernée devrait améliorer pour réduire ces risques. Elle conclut qu'il est possible de se concentrer sur seulement quelques réducteurs de risques. Toutefois, cette stratégie aura plus d'effet sur des acheteurs multicanaux que sur des clients n'utilisant encore le site que pour s'informer.

Mots-clés : commerce électronique, Internet, risque perçu, image du site, réducteurs de risque

\begin{abstract}
This research approaches brakes relative to online shopping, not by trust widely used in the literature, but by perceived risk. Carried out on 1167 loyalty card holders of the Fnac, it identifies first, the main types of perceived risks that penalize online purchases, and second, the website characteristics that should improve the retailer to reduce these risks. It concluded that it is possible to focus on a few risk relievers. However, this strategy will have more effect on multichannel buyers than on customers using the site only for information.
\end{abstract}

Keywords : e-commerce, Internet, perceived risk, website image, risk relievers

${ }^{1}$ L'auteur remercie les équipes de Fnac.com et Fnac SA pour leur participation à cette étude. 


\section{Introduction}

Selon le dernier baromètre de la Fevad (juin 2011), les ventes en ligne de produits et services représentent en France en 2010, 31 milliards d'euros de dépenses, soit une croissance supérieure à $53 \%$ l'an sur les 5 dernières années. De son côté, le panier moyen reste stable autour de 91 euros. Cette progression ininterrompue du commerce électronique français est donc due à deux facteurs : la multiplication du nombre d'internautes $(+128,7 \%$ depuis 2007) et surtout un meilleur taux de transformation des cyber-visiteurs en cyberacheteurs $(72,5 \%$ en 2010 contre 63,3\% en 2007). Toutefois, si $97 \%$ de ceux qui ont expérimenté l'achat en ligne s'en déclarent satisfaits, nombre d'internautes hésitent encore à franchir le pas en raison des risques supplémentaires générés par l'outil Internet en général ou par les caractéristiques de la boutique en ligne. En se référant à la conception dichotomique de Bettman (1973), on parlera dans le premier cas de risque inhérent et dans le second, de risque assumé.

Les e-commerçants n'ont que peu de prise sur le risque inhérent puisque celui-ci est très lié à l'évolution des mentalités vis-à-vis d'Internet (Miyazaki et Fernandez, 2001). En revanche, ils peuvent mieux maîtriser le risque induit par leur propre activité de vente en ligne, afin de transformer davantage de cyber-visiteurs en phase d'information en cyberacheteurs, et ainsi réduire le free-riding (Chau et al., 2007). La réussite de ce pilotage opérationnel du risque implique que chaque enseigne identifie les types de risques perçus par ses consommateurs comme les plus prégnants, puis sélectionne le mix d'attributs du site, le plus efficace pour les réduire (Roselius, 1971). Une telle focalisation sur les risques spécifiques plutôt que sur un risque global permet de mieux cerner les comportements des consommateurs et de préciser les actions à mettre en œuvre (Lee, 2009).

Cette recherche vise donc à répondre à la question suivante : une enseigne de distribution peut-elle réduire le risque d'achat en ligne en se focalisant uniquement sur quelques réducteurs de risque et ce, quels que soient le type de risques et de clients ? Pour y répondre, l'étude est menée sur deux types de clients d'un site marchand (www.fnac.com), les uns n'ayant jamais acheté sur le site (acheteurs exclusifs magasin), les autres achetant tantôt dans les magasins tantôt sur le site de l'enseigne. Après une présentation du cadre conceptuel et de la méthodologie utilisée, elle hiérarchise les types de risques qui pénalisent le plus la vente sur le site choisi, puis repère les leviers d'actions les plus efficaces pour réduire ces risques.

\section{Cadre conceptuel et hypothèses de recherche}

\subsection{L'impact du risque perçu dans le commerce électronique}

Le concept de risque perçu concerne «non seulement ce qui est acquis mais aussi la manière ou l'endroit où c'est acheté » (Hisrich et al., 1972). A ce titre, il servit très tôt à expliquer le refus d'acheter sur les canaux à distance : catalogue ou téléphone (Cox et Rich, 1964). Il fut pourtant, par la suite, peu utilisé pour comprendre le choix des canaux de distribution (Lumpkin et Dunn, 1990). Les recherches sur le commerce électronique lui ont ainsi préféré le concept de confiance (Volle, 1995). Néanmoins pour réduire la résistance des consommateurs envers l'achat en ligne (Forsythe et Shi, 2003), Gefen et al. (2008) préconisent de se concentrer sur le risque perçu. En effet, celui-ci semble plus déterminant encore sur Internet qu'en magasin, pour expliquer la non-transformation des visiteurs en acheteurs (Novak et al., 2000 ; Ko et al., 2004). Compte tenu du rôle majeur joué par le risque perçu entre la phase d'information et la phase d'achat en ligne, il est donc possible de poser l'hypothèse suivante :

H1 - Le risque perçu global diminue davantage l'attitude d'achat en ligne des clients n'achetant jamais sur le site, que de ceux qui achètent tantôt en ligne, tantôt en magasin. 
A la fois miroir (Das et Teng, 2004), antécédent et conséquence de la confiance (Mitchell, 1999), le risque perçu constitue « une variable intermédiaire explicite dans les modèles de traitement de l'information »(Volle, 1995). Depuis Cunningham (1967), il est présenté comme un construit qui combine de manière multiplicative (Bettman, 1973), une composante d'incertitude (probabilité subjective d'occurrence) et une composante de perte potentielle (montant en jeu). Chez les consommateurs, cette incertitude est induite par la méconnaissance de leurs propres besoins, par une certaine incapacité à hiérarchiser leurs choix, ainsi que par un manque de confiance dans leur jugement, couplé à la difficulté de distinguer les attributs les plus prédictifs des objets évalués (Mitchell, 1999). En effet, les individus ne peuvent évaluer toutes les conséquences de leurs actions et les «anticiper avec un haut degré de certitude $\gg$ (Bauer, 1967).

Le risque perçu peut aussi être mesuré directement (Conchar et al., 2004). Il est alors traité, soit comme un construit global (Cases, 2002b), soit comme une variable multidimensionnelle, dont chaque dimension varie en fonction de la situation et du type d'objet considéré (Zikmund et Scott, 1974). Les travaux successifs de Cunningham (1967), Roselius (1971) et de Kaplan et al. (1974) ont ainsi permis de distinguer plusieurs types de risque (tableau 1). Pour une revue de littérature, nous renvoyons à Mitchell (1999).

\begin{tabular}{|l|l|}
\hline \multicolumn{1}{|c|}{ Types de risques } & \multicolumn{1}{c|}{ Définition } \\
\hline Risque financier & $\begin{array}{l}\text { Perte potentielle liée à la défaillance du produit, aux coûts de } \\
\text { réparation élevés ou au sentiment de manque à gagner provoqué } \\
\text { par des produits vendus moins cher ailleurs (risque d'opportunité) }\end{array}$ \\
\hline Risque de performance & Difficulté à juger de la qualité du produit \\
\hline Risque physique & Mise en danger physique en utilisant le produit \\
\hline Risque psychologique & Perte d'estime de soi ou anxiété causée par l'achat du produit \\
\hline Risque social & Perte d'estime de soi causée par les réactions de l'entourage \\
\hline Risque temporel & $\begin{array}{l}\text { Perte de temps lié à la recherche, à l'achat ou à la défaillance du } \\
\text { produit }\end{array}$ \\
\hline
\end{tabular}

Tableau 1 - Principaux types de risque liés à l'achat d'un produit

Dans le domaine du commerce électronique, Forsythe et Shi (2003) placent en tête les mêmes risques que ceux relevés par Mitchell et Harris (2005) pour les points de vente physiques : risque de performance, risque psychologique et risque financier. Certains sont néanmoins plus sensibles sur Internet qu'en magasin (Cases, 2002a ; Laroche et al., 2004 ; Eggert, 2006), en particulier les risques de livraison défectueuse (Cheng et al., 2008) et d'utilisation frauduleuse des données personnelles ou bancaires (Hoffman et al., 1999).

Cependant, à l'exception des risques de performance et temporel, il est impossible de comparer les résultats des études précitées tant certains types de risques mesurés en magasin et sur Internet recouvrent en fonction des auteurs, des acceptions fort différentes. En effet, si les travaux sur les risques perçus en magasin transposent fidèlement les risques associés au produit, ce n'est généralement pas le cas de ceux menés sur l'achat en ligne : par exemple, Forsythe et Shi (2003), Lee (2009) ou Chiu et al. (2011) ne prennent en compte dans le risque financier sur Internet que le risque transactionnel lié à l'utilisation frauduleuse de la carte de paiement et, dans le risque psychologique, le non respect de la vie privée plutôt que le sentiment de perte de contrôle induit par un achat déceptif. Seuls Featherman et Pavlou (2004) conservent, pour ces deux canaux de distribution, une définition identique des concepts.

Par ailleurs, l'importance des risques varie selon que l'on est cyber-visiteur, client intensif ou plus épisodique: le cyber-visiteur accorde plus d'importance aux risques financier, 
psychologique et de perte de temps alors que le client épisodique met surtout l'accent sur le risque de performance (Forsythe et Shi, 2003 ; Ko et al., 2004).

H2 - La combinaison des différents types de risques diminue davantage l'attitude d'achat en ligne des clients n'achetant jamais sur le site, que de ceux qui achètent tantôt en ligne, tantôt en magasin

\subsection{Les caractéristiques d'image du site comme réducteurs des risques spécifiques}

La littérature a davantage étudié les indications susceptibles de réduire un risque relatif au produit acheté (Derbaix, 1983) qu'à son canal d'achat. Les rares recherches axées sur celui-ci concernent essentiellement les magasins. Mitchell et McGoldrick (1996) montrent ainsi que le niveau de prix, l'avis des vendeurs ou la politique de garantie réduisent le risque global d'achat en magasin. De leur côté, Mitchell et Harris (2005) relient les caractéristiques d'image des magasins alimentaires aux différents types de risque: par exemple, la saleté, la localisation du magasin et la difficulté de stationnement au risque physique ; les prix élevés, la faiblesse de l'assortiment ou des garanties au risque financier; l'étroitesse des heures d'ouverture ou la non-assistance des vendeurs au risque de perte de temps et de commodité. Se référant au travail de Glover et Benbasat (2007), Gefen et al. (2008) évoquent trois types d'indications qui impactent l'achat en ligne : des indications fonctionnelles qui conditionnent l'énergie consacrée à l'achat en ligne (temps, argent, effort cognitif), des indications informationnelles centrées sur les risques de fraude durant la transaction, et des indications sur les bénéfices attendus. Si un agent de recommandation extérieur au site permettrait, selon eux, de les optimiser, aucune caractéristique intrinsèque au site n'est identifiée.

Néanmoins, de manière éparse, d'autres recherches indiquent qu'un écart de prix incitatif (Vijayasarathy et Jones, 2000), la manière de présenter l'information (Ko et al., 2004) ou la présence d'un forum de discussion (Lopez-Nicolas et Molina-Castillo, 2008) pourraient réduire les réticences des acheteurs en ligne. En étudiant les effets combinés des réducteurs de risque sur un site fictif, Cases (2002b) montre aussi que la diminution du risque global ne dépend pas de l'accumulation des réducteurs de risque, mais de la mise en avant de garanties : échange ou remboursement du produit, sécurisation des paiements. Ces signaux semblent d'ailleurs avoir plus d'impact en ligne qu'en magasin, surtout pour des produits dominés par des attributs non numériques pour lesquels le risque perçu est supérieur sur Internet (Biswas et Biswas, 2004).

Une telle pénurie de recherches sur les caractéristiques du site susceptibles de réduire le risque d'achat en ligne s'explique surtout par le fait que les sites marchands sont plus souvent analysés en termes de qualité de l'outil (Loiacono et al., 2007 ; Bressolles et Durieu, 2008) que de mix marketing proposé sur le site par l'enseigne (Zhang et al., 2000 ; Rolland et Wallet-Wodka, 2003). La prise en compte de dimensions d'image du site permettrait donc d'identifier de nouveaux réducteurs de risque. Or, les rares travaux qui transposent le concept d'image du magasin (Martineau, 1958) demeurent descriptifs (Spiller et Lohse, 1997 ; Katerattanakul et Siau, 2003 ; Van der Heijden et Verhagen, 2004 ; Chen et Lee, 2005) et n'explorent jamais les relations entre chaque dimension d'image du site et le risque perçu. De plus, même dans ces études, des dimensions susceptibles de limiter le risque perçu sont omises, notamment l'accessibilité du site (référencement) et les conseils qui y sont dispensés. Pourtant, les consommateurs peuvent utiliser certaines caractéristiques de l'image du point de vente pour réduire tel ou tel risque spécifique lié à l'achat en magasin (Mitchell et McGoldrick, 1996; Mitchell et Harris, 2005). Il pourrait en aller de même pour certaines caractéristiques du site dans la mesure où un site marchand peut être décrit à partir des mêmes dimensions d'image qu'un magasin (Volle, 2000 ; Helme-Guizon, 2001 ; Bèzes, 2008). 
H3 - Le nombre et les caractéristiques du site, capables de réduire le risque d'achat en ligne, diffèrent selon la catégorie de risque concernée.

La sensibilité au risque varie également en fonction des caractéristiques personnelles des individus (Finucane et al., 2000 ; Garbarino et Strahilevitz, 2004). Plus le risque est élevé, plus les consommateurs font preuve de « vigilance cognitive » (Pinson, Malhotra et Jain 1984, cités par Volle 1995); ils mobilisent alors un grand nombre de dimensions d'image qui délivrent autant d'indications ou de signaux.

H4 - Le nombre et les caractéristiques du site, capables de réduire le risque d'achat en ligne, diffèrent selon le type de clients (clients n'achetant jamais sur le site; clients achetant tantôt en ligne, tantôt en magasin).

\section{Méthodologie}

\subsection{Terrain et échantillon}

Cette étude a été menée auprès de 1167 adhérents extraits des bases comportementales de la Fnac : 152 acheteurs exclusifs magasin, fréquentant régulièrement le site mais n'ayant acheté depuis 2 ans qu'en magasin, et 1015 acheteurs multicanaux ayant acheté tantôt en magasin tantôt en ligne. Le site Fnac.com a été retenu en raison de sa position sur le secteur des produits techniques, premier univers d'achat des internautes en France et de la possibilité d'accéder aux données comportementales pour segmenter la clientèle.

Le risque d'exclusion (Duffy et al., 2005) étant limité, le questionnaire a été administré en ligne. Conformément aux préconisations d'Ajzen (1991) et pour obtenir des réponses plus homogènes lors de l'évaluation des caractéristiques du site et des types de risques d'achat en ligne, la catégorie de produit a été contrôlée : en concertation avec l'enseigne, un scénario d'achat d'un appareil photo numérique a permis de focaliser l'attention des répondants sur un univers volontairement limité. Des entretiens qualitatifs avaient au préalable, montré que ce produit pouvait être acquis aussi bien en magasin (besoin de se rassurer pendant l'achat) que sur un site marchand (produit à faible budget et à durée de vie relativement brève). Par ailleurs, selon les individus, il pouvait être perçu comme un produit hédonique ou utilitaire, de recherche ou d'expérience.

\subsection{Instruments de mesure utilisés}

\subsubsection{Mesure du risque global perçu et des risques spécifiques}

En nous référant à la distinction de Bettman (1973) entre risque inhérent et risque assumé, nous nous focalisons ici sur le risque assumé d'achat sur le site, et non pas sur le risque propre au produit. En effet, la perception du risque varie selon qu'elle s'applique à un site précis, à la catégorie « site marchand » en général, ou à un produit particulier vendu sur le site.

Comme suggérée par Lumpkin et Dunn (1990) et plutôt que d'agréger divers types de risques qui déstabilisent l'échelle de mesure, une échelle de mesure du risque global a été choisie : celle-ci «n'est plus fondée sur une décomposition du risque à l'aide de ses composantes ou de ses dimensions mais sur une appréhension globale du risque »(Cases, 2002b). L'échelle utilisée, celle de Laroche et al. (2003), présente l'avantage de pouvoir s'appliquer en l'état à tous les canaux de distribution. Le tableau 2 montre que ses qualités psychométriques sont très satisfaisantes : $82,3 \%$ de variance restituée ; $\rho_{\mathrm{j}}=0,813$ et $\rho_{\mathrm{vc}}=0,521$.

\begin{tabular}{|c|l|c|c|}
\hline Items & \multicolumn{1}{|c|}{ Libellé } & Com & Axe1 \\
\hline RISGS1 & $\begin{array}{l}\text { Je suis sûr(e) que j'encourrais un risque si j'achetais un } \\
\text { appareil photo numérique sur le site Fnac.com }\end{array}$ & 0,829 & 0,910 \\
\hline RISGS2 & Tout bien considéré, j'ai le sentiment qu'acheter un appareil & 0,850 & 0,922 \\
\hline
\end{tabular}




\begin{tabular}{|c|l|c|c|}
\hline & $\begin{array}{l}\text { photo numérique sur le site Fnac.com, me causerait beaucoup } \\
\text { d'ennuis en cas de problème }\end{array}$ & & \\
\hline RISGS3 & $\begin{array}{l}\text { Globalement, je suis sûr(e) que je ferais une erreur si } \\
\text { j'achetais un appareil photo numérique sur le site Fnac.com }\end{array}$ & 0,791 & 0,889 \\
\hline $\begin{array}{l}\text { Variance } \\
\text { expliquée }\end{array}$ & & $82,31 \%$ \\
\hline $\boldsymbol{\rho}_{\mathbf{j}}$ & & 0,813 \\
\hline $\boldsymbol{\rho}_{\mathbf{v}}$ & & 0,521 \\
\hline
\end{tabular}

Tableau 2 - Qualité psychométriques de l'échelle de mesure du risque global perçu associé à l'achat sur le site

De leur côté, chaque risque spécifique a été évalué par des mesures mono-item extraites des recherches de Van der Heijden et al. (2003), et Stone et Gronhaug (1993). Compte tenu de l'importance du risque de livraison dans le commerce électronique (Mick et Fournier, 1998), un item spécifique à ce risque est introduit (tableau 3).

\begin{tabular}{|l|l|}
\hline \multicolumn{1}{|c|}{ Items } & \multicolumn{1}{|c|}{$\begin{array}{c}\text { En achetant un appareil photo numérique sur le site, } \\
\text { je risquerais de : }\end{array}$} \\
\hline Risque financier & perdre de l'argent \\
\hline Risque temporel & perdre mon temps \\
\hline Risque de performance & me tromper sur le choix des produits \\
\hline Risque personnel & me faire voler mes données bancaires ou personnelles \\
\hline $\begin{array}{l}\text { Risque de retrait ou de } \\
\text { livraison }\end{array}$ & $\begin{array}{l}\text { avoir des problèmes de retrait, de livraison ou de retour des } \\
\text { produits }\end{array}$ \\
\hline Risque psychologique & me rendre anxieux \\
\hline
\end{tabular}

Tableau 3 - Mesure mono-item des risques spécifiques

3.2.2. Mesure des caractéristiques d'image du site

A partir des recherches menées auparavant sur l'image des magasins et sur celle du site, neuf caractéristiques de la boutique en ligne ont été prises en compte. Nous ne présentons pas ici en détail les échelles de mesure utilisées, ni la procédure de validation qu'elles ont nécessitées: celle-ci a requis trois types d'analyse qualitative (Bèzes, 2008 ; Bèzes et Belvaux, 2009 ; Bèzes, 2009), deux pré-tests et une analyse factorielle confirmatoire réalisée sur l'échantillon interrogé (Bèzes, 2010). Les qualités psychométriques des échelles de mesure d'image sont très satisfaisantes : $\rho_{\mathrm{j}}>0,8$ et $\rho_{\mathrm{vc}}>0,6$ (tableau 4 ).

\begin{tabular}{|l|l|c|c|}
\hline \multicolumn{1}{|c|}{ Dimensions } & \multicolumn{1}{|c|}{ Définition du construit } & $\mathbf{\rho}_{\mathbf{j}}$ & $\mathbf{\rho}_{\mathbf{v}}$ \\
\hline Offre & $\begin{array}{l}\text { Perception des marques, de la qualité et du choix } \\
\text { de produits proposés sur le site }\end{array}$ & 0,931 & 0,694 \\
\hline Prix & Perception de la compétitivité des prix sur le site & 0,905 & 0,657 \\
\hline Disposition & $\begin{array}{l}\text { Perception de l'apparence, de la lisibilité et de } \\
\text { l'ergonomie du site }\end{array}$ & 0,921 & 0,701 \\
\hline Accessibilité & Perception de la facilité d'accès au site & 0,931 & 0,772 \\
\hline Promotions & $\begin{array}{l}\text { Perception de la fréquence et de la visibilité des } \\
\text { promotions sur le site }\end{array}$ & 0,911 & 0,719 \\
\hline Service après-vente & $\begin{array}{l}\text { Perception de la qualité du service après-vente tel } \\
\text { qu'il apparaît sur le site }\end{array}$ & 0,881 & 0,713 \\
\hline
\end{tabular}




\begin{tabular}{|l|l|c|c|}
\hline Institution & $\begin{array}{l}\text { Perception de la présentation de l'enseigne telle } \\
\text { qu'elle apparaît sur le site }\end{array}$ & 0,900 & 0,751 \\
\hline Conseil & Perception des conseils dispensés sur le site & 0,898 & 0,747 \\
\hline Réputation & $\begin{array}{l}\text { Perception de la réputation de l'enseigne telle } \\
\text { qu'elle est communiquée par le site (sérieux, } \\
\text { fiabilité) }\end{array}$ & 0,964 & 0,899 \\
\hline
\end{tabular}

Tableau 4 - Qualité psychométrique des échelles de mesure d'image du site utilisées

\subsubsection{Mesure de l'attitude d'achat sur le site}

Enfin pour mesurer l'attitude à l'égard de l'achat sur le site, a été retenue l'échelle déjà utilisée par Van der Heijden et al. (2000), et mise au point par Jarvenpaa et al. (2000) pour prédire les comportements d'achat en ligne sur un site spécifique. Ses qualités psychométriques sont excellentes: plus de $91 \%$ de la variance restituée; $\rho_{\mathrm{j}}=0,955$ et $\rho_{\mathrm{vc}}=0,876$ (tableau 5).

\begin{tabular}{|c|l|c|c|}
\hline Items & \multicolumn{1}{|c|}{ Libellé } & Com & Axe1 \\
\hline $\mathbf{A A 1}$ & $\begin{array}{l}\text { J'aime l'idée d'acheter un appareil photo numérique sur le } \\
\text { site Fnac.com }\end{array}$ & 0,915 & 0,957 \\
\hline $\mathbf{A A 2}$ & $\begin{array}{l}\text { Avoir recours au site Fnac.com pour acheter un appareil } \\
\text { photo numérique serait une bonne idée }\end{array}$ & 0,926 & 0,962 \\
\hline $\mathbf{A A 3}$ & $\begin{array}{l}\text { L'idée d'acheter un appareil photo numérique sur le site } \\
\text { Fnac.com est tentante }\end{array}$ & 0,911 & 0,954 \\
\hline $\begin{array}{c}\text { Variance } \\
\text { expliquée }\end{array}$ & & $91,74 \%$ \\
\hline $\boldsymbol{\rho}_{\mathbf{j}}$ & & 0,955 \\
\hline $\mathbf{\rho}_{\mathbf{v c}}$ & & 0,876 \\
\hline
\end{tabular}

Tableau 5 - Qualité psychométrique de l'échelle de mesure de l'attitude d'achat sur le site

L'ensemble de ces construits ont été mesurés à l'aide d'échelles d'intervalle de type Likert à 7 points et à choix non forcé.

\section{Test des hypothèses et discussion}

La première hypothèse pose que le risque global perçu influence davantage l'attitude d'achat en ligne des clients qui n'achètent jamais sur le site. Une régression linéaire simple montre que le risque perçu dégrade l'attitude d'achat en ligne des deux groupes d'acheteurs (tableau 6). Mais contrairement au présupposé théorique, une comparaison des $\mathrm{R}^{2}$ ajustés et des coefficients de régression met en évidence une dégradation plus forte de l'attitude chez les acheteurs multicanaux que chez les acheteurs exclusifs magasin; selon le test de Chow (1960), cet écart est significatif : rejet de l'hypothèse de stabilité des paramètres $(\mathrm{p}<0,05)$. H1 est rejetée.

\begin{tabular}{|l|c|c|}
\hline $\begin{array}{c}\text { Coefficients de régression standardisés } \\
(* * * \mathrm{p}<0,000 ; * * \mathrm{p}<0,01)\end{array}$ & $\begin{array}{c}\text { Acheteurs exclusifs } \\
\text { magasin }\end{array}$ & $\begin{array}{c}\text { Acheteurs } \\
\text { multicanaux }\end{array}$ \\
\hline Risque global => Attitude achat site & $-0,236 * *$ & $-0,436 * * *$ \\
\hline $\mathbf{R}^{2}$ ajusté & $\mathbf{0 , 0 4 9}$ & $\mathbf{0 , 1 8 9}$ \\
\hline Test de Chow & \multicolumn{2}{|c|}{$149,84>3 \mathrm{~F}(2,1063)$} \\
\hline
\end{tabular}

Tableau 6 - Effet du risque global sur l'attitude d'achat sur le site par types de clients 
Ce résultat contre-intuitif pourrait s'expliquer par la seule prise en compte du risque et de l'attitude associés à l'achat sur un site spécifique, indépendamment du risque et de l'attitude inhérents au canal Internet en général (assumé vs inhérent). En effet, un test du Khideux mené sur l'attitude inhérente au canal d'achat conclut que les clients de l'enseigne qui n'achètent qu'en magasin sont également moins ouverts que les autres à l'idée d'acheter sur Internet ; par ailleurs, ils perçoivent l'achat sur le site Fnac.com comme plus risqué $(F=11,118, p<0,001)$.

Cette aversion générique vis-à-vis de l'achat sur Internet serait donc en mesure de modérer la relation entre le risque et l'attitude assumés. Néanmoins, une série de régressions linéaires menées sur des groupes d'acheteurs diversement prédisposés vis-à-vis de l'achat sur Internet, démontre le contraire. D'autres raisons plausibles peuvent alors être invoquées : par exemple, le fait que les acheteurs exclusifs magasin se censurent $a$ priori pour acheter en ligne, ou un écart de sensibilité au risque selon les types d'acheteurs. En l'absence de données collectées sur ces variables individuelles, il est impossible de conclure ici.

La deuxième hypothèse suppose que la combinaison des différents types de risques a plus d'incidence sur l'attitude d'achat en ligne de ceux n'achetant jamais sur le site que de ceux qui le font de temps à autre. Le tableau 7 infirme, là aussi, cette hypothèse. $\mathrm{H} 2$ est rejetée.

\begin{tabular}{|l|c|c|}
\hline $\begin{array}{c}\text { Coefficients de régression standardisés } \\
(* * * \mathrm{p}<0,000 ; * * \mathrm{p}<0,01 ; * \mathrm{p}<0,05)\end{array}$ & $\begin{array}{c}\text { Acheteurs exclusifs } \\
\text { magasin }\end{array}$ & $\begin{array}{c}\text { Acheteurs } \\
\text { multicanaux }\end{array}$ \\
\hline Risque financier & & $-0,057$ \\
\hline Risque temporel & & $-0,138 * * *$ \\
\hline Risque de performance & $-0,374 * * *$ & $-0,162 * * *$ \\
\hline Risque personnel & & 0,064 \\
\hline Risque de retrait ou de livraison & & $-0,137 * *$ \\
\hline Risque psychologique & & $-0,091 *$ \\
\hline $\mathbf{R}^{2}$ ajusté & $\mathbf{0 , 1 3 5}$ & $\mathbf{0 , 1 7 7}$ \\
\hline
\end{tabular}

Tableau 7 - Effets des types de risques sur l'attitude à l'égard de l'achat sur le site

Ce rejet est cohérent par rapport à celui de l'hypothèse H1. Les acheteurs multicanaux étant plus concernés par un éventuel achat en ligne et plus influencés par le risque global perçu, ils sont aussi amenés à examiner davantage de risques spécifiques. Outre la crainte de ne pouvoir évaluer convenablement les qualités du produit (risque de performance partagé avec les clients n'achetant qu'en magasin), les acheteurs multicanaux sont également très influencés par deux autres types de risques: celui de perdre du temps dans le processus d'achat du produit (risque temporel) et celui de subir des déconvenues lors de la livraison ou du retour des articles achetés.

Le fait d'avoir opposé des acheteurs multicanaux à des clients habitués à s'informer sur le site Fnac.com (77 \% le visitent au moins 1 fois par mois) mais n'achetant en définitive que dans les magasins de l'enseigne, permet d'affiner les apports de Forsythe et Shi (2003) ou de Ko et al. (2004). En effet, bien que le risque de performance demeure pour tous, le facteur le plus dissuasif envers l'achat en ligne, le risque temporel n'est pris en compte que par ceux qui doivent vraiment arbitrer entre l'achat sur le site et celui en magasin: les acheteurs multicanaux. Il en va de même du risque de livraison, de retrait ou de retour des produits, ce qui confirme et précise les résultats de Cheng et al. (2008), mais aussi ceux de la Fevad.

Remarquons encore deux autres points : d'une part, l'absence d'influence du risque de vol des données personnelles ou bancaires (risque personnel) pourrait s'expliquer par l'image de la Fnac (capital-confiance, antériorité de sa boutique en ligne), et surtout par l'évolution des 
mentalités (Hoffman et al., 1999 ; Miyazaki et Fernandez, 2001) ; d'autre part, le caractère anecdotique du risque financier peut être dû à la volonté de nous conformer à la définition initiale de ce concept (Stone et Gronhaug 1993 ou Featherman et Pavlou 2004 vs Forsythe et Shi 2003), mais aussi à une moindre sensibilité aux écarts de prix pour les adhérents Fnac.

Les deux dernières hypothèses concernent les caractéristiques du site susceptibles de réduire le risque d'achat en ligne. Pour faciliter l'analyse et plutôt que de présenter en détail les résultats des régressions linéaires multiples, le tableau 8 hiérarchise les attributs du site qui réduisent significativement les différents risques selon le type d'acheteurs; les rangs soulignés correspondent à ceux observés chez les acheteurs multicanaux, les autres chez les acheteurs exclusifs magasin.

\begin{tabular}{|c|c|c|c|c|c|c|}
\hline & $\begin{array}{c}\text { Risque de } \\
\text { performance }\end{array}$ & $\begin{array}{c}\text { Risque } \\
\text { temporel }\end{array}$ & $\begin{array}{c}\text { Risque } \\
\text { de } \\
\text { livraison }\end{array}$ & $\begin{array}{c}\text { Risque } \\
\text { psychologique }\end{array}$ & $\begin{array}{l}\text { Risque } \\
\text { financier }\end{array}$ & $\begin{array}{c}\text { Risque } \\
\text { personnel }\end{array}$ \\
\hline Accessibilité & & 1 et $\underline{1}$ & & $\underline{4}$ & 2 et $\underline{3}$ & $\underline{2}$ \\
\hline Conseil & 2 et $\underline{1}$ & 2 et $\underline{4}$ & $\underline{5}$ & $\underline{5}$ & & \\
\hline Disposition & $\underline{4}$ & & $\underline{2}$ & $\underline{2}$ & $\underline{4}$ & 2 \\
\hline Institution & & & + & + & & + \\
\hline Offre & 1 et 2 & $\underline{2}$ & 3 et $\underline{4}$ & 2 & & $\underline{1}$ \\
\hline Prix & + & & & & 1 et $\underline{1}$ & + \\
\hline Promotions & & & & & & 3 \\
\hline Réputation & $\underline{3}$ & 3 et $\underline{3}$ & 2 et $\underline{3}$ & 1 et $\underline{3}$ & $\underline{2}$ & $\underline{4}$ \\
\hline $\begin{array}{l}\text { Service } \\
\text { après-vente }\end{array}$ & & $\underline{5}$ & 1 et 1 & 3 et $\underline{1}$ & & 1 et $\underline{3}$ \\
\hline
\end{tabular}

Tableau 8 - Hiérarchisation des réducteurs de risque par types de risques et d'acheteurs

Trois dimensions de l'image du site influent peu sur chaque type de risque perçu. C'est le cas de la mise en avant de l'histoire et des valeurs de l'institution et ce, quel que soit le type d'acheteurs ; le signe positif figurant tableau 8 est à relativiser puisque cet effet n'est constaté que lorsque cette variable est combinée à d'autres dans la perception des individus.

De leur côté et contrairement à ce que relèvent Vijayasarathy et Jones (2000), des prix avantageux ne limitent que le risque financier et ce, pour tous les types d'acheteurs; dans le cas des risques de performance et personnel, ils pourraient en revanche avoir un effet inflationniste lorsqu'ils ne sont plus considérés isolément tel un repère périphérique (Petty et al., 1983), mais combinés à d'autres critères.

Enfin, l'intensification des promotions sur le site n'a pas d'effet réducteur, hormis sur le risque personnel pour les acheteurs exclusifs magasin, comme si elle compensait le prix du danger que constitue pour eux, l'acte d'acheter en ligne. Bien qu'en général, les promotions soient davantage considérées comme une source de valeur perçue que de risque, leur absence d'effet sur le risque financier est surprenante. Le fait que les adhérents Fnac soient loin d'être des chasseurs de prix et de bonnes affaires, pourrait expliquer ce résultat.

A l'inverse, toutes les autres caractéristiques du site réduisent significativement la plupart des risques spécifiques analysés, au moins pour certains types d'acheteurs. C'est le cas de la réputation de l'enseigne (confiance, sérieux, fiabilité) telle qu'elle est communiquée par le site. Celle-ci figure parmi les trois premiers réducteurs de chaque risque spécifique, quasiment toujours pour les acheteurs multicanaux, souvent pour les acheteurs exclusifs magasin. 
L'offre proposée par le site constitue aussi l'un des premiers réducteurs de risques (risques de performance et temporel). Une régression linéaire à partir des items constitutifs du construit « offre », montre que la certitude de pouvoir acheter en ligne de grandes marques et des produits adaptés à ses besoins a plus d'influence que la qualité ou la sélection des produits.

L'image du service après-vente transmise par le site peut être également considérée comme un réducteur majeur de risque, en particulier du risque de livraison. Le rôle joué par ces trois caractéristiques du site confirme les conclusions de Roselius (1971) dans le monde de la distribution physique; ceci complète, dans le domaine du commerce électronique, les résultats de Chen et Dubinsky (2003), trop focalisés sur la réputation du e-commerçant (vs du site) pour réussir à le prouver.

Trois autres éléments de la boutique en ligne contribuent encore à réduire le risque d'achat : son accessibilité (référencement) qui limite principalement les coûts de recherche des informations (risque temporel) ; sa disposition (atmosphère, facilité de navigation, lisibilité) qui rassure le client sur le risque de livraison mais aussi sur la sécurité de ses données personnelles; les conseils délivrés sur le site qui ont un effet considérable sur le risque de performance et, dans une moindre mesure, sur le risque temporel.

L'analyse précédente permet donc d'accepter l'hypothèse selon laquelle le nombre et les caractéristiques du site, susceptibles de réduire le risque d'achat en ligne, diffèrent selon la catégorie de risque concernée. Il semble toutefois possible de se focaliser sur quelques éléments du site pour réduire la plupart des risques majeurs. H3 est validée.

De même, le nombre et les caractéristiques du site mobilisées pour chaque type de risque varient selon le type de clients ; les acheteurs multicanaux semblent prendre en considération davantage de réducteurs de risque, vraisemblablement parce qu'ils sont potentiellement plus ouverts à l'idée d'acheter en ligne. H4 est validée.

Le tableau 9 synthétise les résultats des hypothèses testées.

\begin{tabular}{|l|c|}
\hline Libellés des hypothèses & Décision \\
\hline $\begin{array}{l}\text { H1 - Le risque global diminue davantage l'attitude d'achat en ligne des } \\
\text { clients n'achetant jamais sur le site que de ceux qui achètent tantôt en ligne, } \\
\text { tantôt en magasin. }\end{array}$ & Rejetée \\
\hline $\begin{array}{l}\text { H2 - La combinaison des différents types de risques diminue davantage } \\
\text { l'attitude d'achat en ligne des clients n'achetant jamais sur le site que de } \\
\text { ceux qui achètent tantôt en ligne, tantôt en magasin }\end{array}$ & Rejetée \\
\hline $\begin{array}{l}\text { H3 - Le nombre et les caractéristiques du site, capables de réduire le risque } \\
\text { d'achat en ligne, diffèrent selon la catégorie de risque concernée. }\end{array}$ & Validée \\
\hline $\begin{array}{l}\text { H4 - Le nombre et les caractéristiques du site, capables de réduire le risque } \\
\text { d'achat en ligne, diffèrent selon le type de clients (clients n'achetant jamais } \\
\text { sur le site, clients achetant tantôt en ligne, tantôt en magasin). }\end{array}$ & Validée \\
\hline
\end{tabular}

Tableau 9 - Récapitulatif des hypothèses testées

\section{Conclusion}

Cette recherche partait d'une problématique managériale simple, mais encore peu étudiée : pour réduire le risque perçu, réputé constituer un obstacle majeur au développement de la vente en ligne, un e-commerçant peut-il concentrer son action sur quelques réducteurs de risque, quels que soient le type de risques et de clients concernés? Pour y répondre, l'étude a analysé les risques spécifiques générés par un site réel, et non par tel ou tel produit qui y est 
acheté ; ceci la distingue de travaux pionniers, menés sur des sites expérimentaux (Cases 2002a ; Cases 2002b) ou sur le risque inhérent au canal Internet (Forsythe et Shi, 2003).

Il apparaît nécessaire de réduire en priorité trois risques : d'abord, le risque de performance qui affecte tous les clients et concerne l'incertitude sur le produit, mais aussi sur le processus d'achat (Liang et Huang, 1998) ; ensuite pour les acheteurs multicanaux, le risque de perte de temps et celui de livraison. Ces résultats concordent avec la littérature et semblent généralisables à d'autres sites marchands, pour d'autres produits tangibles. En revanche, bien que fréquemment cité, le risque de perte des données bancaires ou personnelles n'a pas d'incidence fâcheuse. Ceci pourrait avoir été favorisé par le choix d'une enseigne à forte notoriété, exploitant un site qui bénéficie d'une longue antériorité.

Pour réduire ces risques spécifiques, l'enseigne concernée aurait alors intérêt à porter ses efforts sur les éléments fonctionnels de sa boutique en ligne, et en premier lieu, sur l'image de sérieux et de fiabilité (réputation), mais aussi sur celle du service après-vente. Ceci implique une clarté et une repérabilité immédiate des informations relatives aux garanties (Cases, 2002b), aux conditions de reprise des produits ainsi qu'aux délais de livraison. Plutôt que d'activer un lien "conditions générales de vente», souvent noyé au milieu des informations légales, il conviendrait notamment d'insérer en bonne place sur la page d'accueil, un encart au message explicite, quitte à sacrifier la mise en avant d'une promotion. En effet, si les promotions génèrent un supplément de trafic de visiteurs (Bèzes et Belvaux, 2009), elles ne contribuent guère à réduire le risque d'achat, donc à transformer des cybervisiteurs en cyberacheteurs.

Cet accent mis sur les attributs fonctionnels pourrait corroborer l'idée qu'un site marchand devrait être impérativement utile, informatif et clair, mais pas nécessairement divertissant ou imaginatif (Chen et al., 2002 ; Rolland et Wallet-Wodka, 2003 ; Elliott et Speck, 2005). Par ailleurs, comme le recommandent Novak et al. (2000), les webdesigners ne devraient pas seulement chercher à stimuler les internautes les plus avides d'expériences, par une surenchère de mises en scène ; ils devraient également respecter les exigences de facilité d'usage et de navigation des internautes centrés sur un but. Ce constat pourrait enfin étayer l'idée évoquée par la littérature, qu'un site Internet développe un type de traitement de l'information plus analytique qu'intuitif, le côté hédonique apparaissant en sus.

L'enseigne étudiée devrait aussi valoriser le plus possible l'offre proposée, en insistant sur la certitude de trouver sur son site, un choix complet de grandes marques et de produits sélectionnés, accompagnés de conseils pertinents. Enfin, un meilleur référencement du site constitue non seulement un gage de création de trafic, mais aussi de réduction du risque temporel. Seulement dans un second temps, de nouvelles affordances virtuelles (Li et al., 2003), par exemple la réalité augmentée, pourraient continuer à réduire le risque de performance qui constitue le frein majeur à l'achat en ligne, au moins dans le cas du produit retenu pour cette étude.

Il est néanmoins probable que ces améliorations porteront essentiellement leurs fruits sur les clients ayant déjà acheté sur le site. En effet, ce sont eux qui prennent en compte de manière analytique, le plus de risques spécifiques et le plus de réducteurs de risques. Il semble donc plus réaliste de vouloir transformer des acheteurs épisodiques en acheteurs intensifs que des cyber-visiteurs en cyberacheteurs. Quant aux clients qui s'informent en ligne mais continuent de n'acheter qu'en magasin, leurs motifs de non-achat semblent être moins liés au risque généré par le site, qu'à d'autres caractéristiques personnelles ou situationnelles.

Malgré ses apports, cette recherche présente plusieurs limites, qui l'empêchent d'être généralisée. La première tient au terrain choisi. Le site analysé existe depuis plus de dix ans et 
bénéficie de l'excellente image de l'enseigne Fnac, ce qui peut limiter le risque d'achat en ligne mais aussi la portée de l'image du site par rapport à la réputation générale du détaillant (Lwin et Williams 2006 ; Biswas et Biswas 2004).

Une autre limite concerne la catégorie de produit servant de support à l'évaluation du risque induit par le canal d'achat. Avoir retenu un appareil photo numérique, produit technologiquement complexe et acheté sporadiquement, pourrait avoir renforcé la prépondérance du risque de performance (Biswas et Biswas, 2004 ; Bhatnagar et al., 2000). De plus, il aurait été utile d'évaluer en parallèle le risque associé à ce produit afin de mesurer le risque supplémentaire effectivement induit par l'usage du canal.

Une dernière limite tient au fait de ne pas avoir utilisé des échelles multi-items de mesure pour chaque risque spécifique; celles-ci auraient eu pour avantages de combiner les spécificités de chaque item autour d'une moyenne, de discriminer plus précisément les individus et ainsi de réduire les erreurs de mesure (Churchill, 1979).

Outre une réplication de l'étude sur d'autres sites marchands, deux voies de recherche sont donc envisageables. Il s'agirait tout d'abord, d'introduire le risque perçu dans un réseau de causalités plus larges prenant en compte non seulement l'image du site mais aussi d'autres variables qui interviennent dans le processus de choix du canal d'achat, comme la valeur perçue. Cette relativisation du risque perçu permettrait de préciser la recherche de Forsythe et al. (2006) selon laquelle le risque perd de l'importance face à la valeur perçue lorsque l'expérience en ligne des cyberacheteurs s'accroît ; elle pourrait également démontrer qu'il est plus aisé d'améliorer la valeur perçue que de réduire le risque d'achat en ligne (Lee, 2009).

Une seconde piste consisterait à prendre en compte les variables personnelles susceptibles d'expliquer les raisons de non-achat des acheteurs exclusifs magasin.

\section{Bibliographie}

Ajzen I. (1991), "The theory of planned behaviour", Organizational Behavior and Human Processes, Vol. 50, p. 179-211.

Bhatnagar A., Misra S. et Rao R. (2000), "On risk, convenience, and Internet shopping behavior", Communications of the ACM, Vol. 43, No. 11, p. 98-105.

Bauer R. (1967), "Consumer behavior as risk taking", in D. F. Cox (cood.), Risk taking and information handling in consumer behaviour, Cambridge, Mass., Harvard University Press, p. 23-33.

Bettman J. (1973), "Perceived risk and its components: a model and empirical", Journal of Marketing Research, Vol. 10, No. 2, p. 184-190.

Bèzes C. (2008), «La cohérence d'image dans le cas d'une extension du système de distribution : définition d'une grille d'analyse appliquée aux canaux de distribution de produits high-tech », 11ème Colloque Etienne Thil, p. 1-26.

Bèzes C. et Belvaux B. (2009), «Typologie de sites web : une approche par l'image transmise », $28^{e ̀ m e}$ Conférence Internationale de Management Stratégique AIMS, p. 1-20.

Bèzes C. (2009), "Une exploration qualitative des dimensions d'image de deux sites marchands français », $8^{\text {ème }}$ Journée du e-marketing, p. 1-39.

Bèzes C. (2010), «La congruence perçue des magasins et du site Internet : effets sur le choix du canal d'achat - Le cas de la Fnac », Thèse de Doctorat en Sciences de Gestion, p. 1-519.

Bhatnagar A., Misra S. et Rao R. (2000), "On risk, convenience, and Internet shopping behavior", Communications of the ACM, Vol. 43, No. 11, p. 98-105.

Biswas D. et Biswas A. (2004), "The diagnostic role of signals in the context of perceived risks in online shopping: do signals matter more on the web?", Journal of Interactive Marketing, Vol. 18, No. 3, p. 30-45. 
Bressolles G. et Durieu B. (2008), «L'impact de la qualité de service électronique sur la satisfaction et les intentions de fidélité : différence entre acheteurs et visiteurs », Actes $d u$ $24^{\text {ème }}$ Congrès International de l'Association Française de Marketing, p. 1-23.

Cases A-S. (2002a), "Perceived risk and risk-reduction strategies in Internet shopping", International Review of Retail, Distribution and Consumer Research, Vol. 12, No. 4, p. 375394.

Cases A-S. (2002b), «Effets des combinaisons de réducteurs sur le risque perçu dans le contexte de l'achat électronique sur Internet », Actes du $18^{\text {ème }}$ Congrès International de l'Association Française de Marketing, Lille, p. 273-286.

Chau P., Hu P., Lee B. et Au A. (2007), "Examining customers' trust in online vendors and their dropout decisions: an empirical study", Electronic Commerce Research and Applications, Vol. 6, p. 171-182

Chen Q., Clifford S. et Wells W. (2002), "Attitude toward the site II: new information", Journal of Advertising Research, mars-avril, p. 33-45.

Chen Z. et Dubinsky A. (2003), "A conceptual model of perceived customer value in ecommerce: a preliminary investigation", Psychology \& Marketing, Vol. 20, No. 4, p. 323-347. Chen W-J. et Lee C. (2005), "The impact of web site image and consumer personality on consumer behaviour", International Journal of Management, Vol. 22, No. 3, p. 484-496.

Cheng J., Wang E., Lin J, Chen L. et Huang W. (2008), "Do extrinsic cues affect purchase risk at international e-tailers: the mediating effect of perceived e-tailer service quality", Journal of Retailing \& Consumer Services, Vol. 15, No. 5, p. 420-428.

Chiu H-C., Hsieh Y-C., Roan J., Tseng K-J. et Hsieh J-K. (2011), "The challenge for multichannel services: cross-channel free-riding behavior", Electronic Commerce Research and Applications, Vol. 10, No. 2, p. 268-277.

Chow G. (1960), "Tests of equality between sets of coefficient in two linear regressions", Econometrica, Vol. 28, No. 3, p. 591-605.

Churchill G. (1979), "A paradigm for developing better measures of marketing constructs", Journal of Marketing Research, Vol. 16, p. 64-73.

Conchar M., Zinkhan G., Peters C. et Olavarrieta S. (2004), "An integrated framework for the conceptualization of consumers' perceived-risk processing", Journal of the Academy of Marketing Science, Vol. 32, No. 4, p. 418-436.

Cox D. et Rich S. (1964), "Perceived risk and consumer decision-making - the case of the telephone shopping", Journal of Marketing Research, Vol. 1, No. 4, p. 32-39.

Cunningham, S. M. (1967). "Perceived risk and brand loyalty" in D. F. Cox (cood.). RiskTaking and Information Handling in Consumer Behavior. Boston, MA: Boston University Press, p. 507-523.

Das T. et Teng B-S. (2004), "The risk-based view of trust: a conceptual framework", Journal of Business and Psychology, Vol. 19, No. 1, p. 85-116.

Derbaix C. (1983), "Perceived risk and risk relievers: an empirical investigation", Journal of Economic Psychology, Vol. 3, p. 19-38.

Duffy B., Smith K., Terhanian G. et Bremer J. (2005), "Comparing data from online and faceto-face surveys", International Journal of Market Research, Vol. 47, No. 5, p. 615-639.

Eggert A. (2006), "Intangibility and perceived risk in online environments", Journal of Marketing Management, Vol. 22, No. 5/6, p. 553-572.

Elliott M. et Speck P. (2005), "Factors that affect attitude toward a retail web site", Journal of Marketing Theory \& Practice, Vol. 13, No. 1, p. 40-51.

Featherman M. et Pavlou P. (2008), "Predicting e-services adoption: a perceived risk facets perspective”, International Journal of Human-Computer Studies, Vol. 59, p. 451-474.

Finucane M., Slovic P., Mertz C., Flynn J. et Satterfield T. (2000), "Gender, race, and perceived risk: the "white male" effect", Health, Risk \& Society, Vol. 2, No. 2, p. 159-172 
Forsythe S., Liu C., Shannon D. et Gardner L. (2006), "Development of a scale to measure the perceived benefits and risks of online shopping", Journal of Interactive Marketing, Vol. 20, No. 2, p.55-75.

Forsythe S. et Shi B. (2003), "Consumer patronage and risk perceptions in Internet shopping", Journal of Business Research, Vol. 56, p. 867-875.

Garbarino E. et Strahilevitz M. (2004), "Gender differences in the perceived risk of buying online and the effects of receiving a site recommendation", Journal of Business Research, Vol. 57, No. 7, p. 768-775.

Gefen D., Benbasat I. et Pavlou P. (2008), "A research agenda for trust in online environment", Journal of Management Information Systems, , Vol. 24, No. 4, p. 275-286.

Helme-Guizon A. (2001), « Le comportement du consommateur sur un site marchand est-il fondamentalement différent de son comportement en magasin ? Proposition d'un cadre d'appréhension de ses spécificités », Recherche et Applications en Marketing, Vol. 16, No. 3, p. 25-38.

Hisrich R., Dornoff R. et Kernan J. (1972), "Perceived risk in store selection", Journal of Marketing Research, Vol. 9, p. 435-439.

Hoffman D., Novak T. et Peralta M. (1999), "Building consumer trust online", Communications of the ACM, Vol. 42, No. 4, p. 80-85.

Jarvenpaa S. et Tractinsky N. (1999), "Consumer trust in an internet store: a cross-cultural validation", Journal of Computer-Mediated Communication, Vol. 5, No. 2, p. 1-34.

Kaplan L., Szybillo G. et Jacoby J. (1974), "Components of perceived risk in product purchase: a cross-validation", Journal of Applied Psychology, Vol. 59, No. 3, p. 287-291.

Katerattanakul P. et Siau K. (2003), "Creating a virtual store image", Communications of the ACM, Vol. 46, No. 12, p. 226-232.

Ko H., Jung J., Kim J-Y. et Shim S., (2004), "Cross-cultural differences in perceived risk of online shopping", Journal of Interactive Advertising, Vol.4, No. 2, p.1-14.

Laroche M., Bergeron J. et Goutaland C. (2003), "How intangibility affects perceived risk: the moderating role of knowledge and involvement", Journal of Services Marketing, Vol. 17, No. 2, p. 122-140.

Laroche M., McDougall G., Bergeron J. et Zhiyong Y. (2004), "Exploring how intangibility affects perceived risk", Journal of Service Research, Vol. 6, No. 4, p. 373-389.

Lee (2009), "Factors influencing the adoption of internet banking: an integration of TAM and TPB with perceived risk and perceived benefit", Electronic Commerce Research and Applications, Vol. 18, No. 3, p. 130-141.

Li H., Daugherty T. et Biocca F. (2003), "The role of virtual experience in consumer learning", Journal of Consumer Psychology, Vol. 13, N 4, p. 395-407.

Liang T-P. et Huang J-S. (1998), “An empirical study on consumer acceptance of products in electronic markets: a transaction cost model", Decision Support Systems, Vol. 24, p. 29-43.

Loiacono E., Watson R. et Goodhue D. (2007), "WebQual: an instrument for consumer evaluation of web sites", International Journal of Electronic Commerce, Vol. 11, No. 3, p. 51-87.

Lopez-Nicolas C. et Molina-Castillo F. (2008), "Customer knowledge management and ecommerce: the role of customer perceived risk", International Journal of Information Management, Vol. 28, No. 2, p. 102-113.

Lumpkin J. et Dunn M. (1990), "Perceived risk as a factor in store choice: an examination of inherent versus handled risk", Journal of Applied Business Research, Vol. 6, No. 2, p; 104118.

Lwin M. et Williams J. (2006), "Promises, promises: how consumers respond to warranties in internet retailing", Journal of Consumer Affairs, Vol. 40, No. 2, p. 236-259. 
Martineau P. (1958), "The personality of the retail store", Harvard Business Review, Vol. 36, No. 1, p. 47-55.

Mick D. et Fournier S. (1998), "Paradoxes of technology: consumer cognizance, emotions, and coping strategies", Journal of Consumer Research, Vol. 25, p. 123-143.

Mitchell V. (1999), "Consumer perceived risk: conceptualisations and models", European Journal of Marketing, Vol. 33, No. 1/2, p. 163-195.

Mitchell V. et McGoldrick P. (1996), "Consumers' risk-reduction strategies: a review and synthesis", International Review of Retail, Distribution \& Consumer Research, Vol. 6, No. 1, p. $1-33$.

Mitchell V-W. et Harris G. (2005), "The importance of consumers' perceived risk in retail strategy", European Journal of Marketing, Vol. 90, No. 7/8, p. 821-837.

Miyazaki A. et Fernandez A. (2001), "Consumer perceptions of privacy and security risks for online shopping", Journal of Consumer Affairs, Vol. 35, No. 1, p. 27-44.

Novak T., Hoffman D. et Yung Y-F. (2000), "Measuring the customer experience in online environments: a structural modeling approach", Marketing Science, Vol. 19, No. 1, p. 22-42.

Petty R., Cacioppo J. et Schumann D. (1983), "Central and peripheral routes to advertising effectiveness: the moderating role of involvement", Journal of Consumer Research, Vol. 10, p. 135-146.

Rolland S. et Wallet-Wodka D. (2003), «La qualité des sites web marchands en distribution : proposition d'une échelle de mesure e-qual», Actes du 6ème colloque Etienne Thil, La Rochelle, p. 1-21.

Roselius T. (1971), "Consumer rankings of risk reduction methods", Journal of Marketing, Vol. 35, p. 56-61.

Spiller P. et Lohse G. (1997), “A classification of Internet retail stores”, International Journal of Electronic Commerce, Vol. 6, No. 2, p. 29-56.

Stone R. et Gronhaug K. (1993), "Perceived risk: further considerations for the marketing discipline", European Journal of Marketing, Vol. 27, No. 3, p. 39-50.

Van der Heijden H., Verhagen T. et Creemers M. (2000), "Predicting online purchase behavior: replications and tests of competing models", Serie Research Memoranda Faculteit der Economische Wetenschappen en Econometric, p. 1-64.

Van der Heijden H., Verhagen T. et Creemers M. (2003), "Understanding online purchase intentions: contributions from technology and trust perspectives", European Journal of Information Systems, Vol. 12, No. 1, p. 41-48.

Van der Heijden H. et Verhagen T. (2004), "Online store image: conceptual foundations and empirical measurement", Information \& Management, Vol. 41, p. 609-617.

Vijayasarathy L. et Jones J. (2000), "Print and Internet catalog shopping : assessing attitudes and intentions", Internet Research: Electronic Networking Applications and Policy, Vol. 10, No. 3, p. 191-202.

Volle P. (1995), «Le concept de risque perçu en psychologie du consommateur : antécédents et statut théorique », Recherche et Applications en Marketing, Vol. 10, No. 1, p. 39-56.

Volle P. (2000), «Du marketing des points de vente à celui des sites marchands : spécificités, opportunités et questions de recherche », Revue Française de Marketing, Vol. 177/178, p. 83100.

Zhang X., Keeling K. et Pavur R. (2000), "Information quality of commercial web site home pages: an explorative analysis", Proceedings of the 21th ICIS, Brisbane, p. 164-175

Zikmund W. et Scott J. (1974), "A multivariate analysis of perceived risk and self-confidence and information sources", Advances in Consumer Research, Vol. 1, No. 1, p. 406-416. 